\title{
A Wideband Linear-to-Circular Polarizer based on Hexagonal FSS on PDMS Substrate for CubeSats
}

\author{
Hidayath Mirza \\ Dept. of Electrical Engineering \\ College of Engineering, \\ Jazan University \\ Jazan, Kingdom of Saudi Arabia \\ ORCID ID: 0000-0002-9050-264X \\ Toufiq M Hossain \\ School of Engineering and Information \\ Technology, University of New South \\ Wales, Canberra \\ Campbell, ACT 2600, Australia. \\ toufiq_md.hossain@adfa.edu.au
}

\author{
Ping Jack Soh \\ Centre for Wireless Communications, \\ University of Oulu, P.O. Box 4500, \\ FI-90014 Oulu, Finland \\ pingjack.soh@oulu.fi
}

Sen Yan

School of Information and

Communications Engineering,

$X i$ 'an Jiaotong University,

Xi'an 710049, China

sen.yan@xjtu.edu.cn

\author{
Rais Ahmad Sheikh \\ Dept. of Electrical Engineering \\ College of Engineering \\ Jazan University \\ Jazan, Kingdom of Saudi Arabia. \\ raisahmadlko@gmail.com \\ Jiahao Zhang, Fengyi Luo \\ National Key Laboratory of Science \\ and Technology on Vessel Integrated \\ Power System, Naval University of \\ Engineering, Wuhan, China \\ jiahao.z@hotmail.com, \\ 635645845@qq.com
}

\begin{abstract}
A novel flexible single-layered and single-sided linear to-circular polarizer is proposed in this work. The proposed polarizer is designed for a deployable format for application in a $3 \mathrm{U}$ Cube Satellites. It features a near-zero $3 \mathrm{~dB}$ $A R$, with a size of $0.22 \lambda_{0} \times 0.27 \lambda_{0} \times 0.03 \lambda_{0}$ and a $3 \mathrm{~dB} A R$ fractional bandwidth of $34.39 \%$, starting from $1.95 \mathrm{GHz}$ to 2.76 GHz. It performs with a conversion efficiency bandwidth covering $90 \%$ of $43.47 \%$, starting from $1.8 \mathrm{GHz}$ to $2.8 \mathrm{GHz}$. This indicates the polarizer's wideband $\mathrm{CP}$ performance, with a pure $\mathrm{CP}$ centered at $2.41 \mathrm{GHz}$.
\end{abstract}

Keywords- Polarizers, frequency selective surfaces, metamaterials, CubeSats.

\section{INTRODUCTION}

Polydimethylsiloxane (PDMS) is a polymer which has found vast applications in electronic devices when used as a substrate. This is due to its versatile properties such as: lightweight, flexible nature, antistatic and have resistant against abrasion and chemicals, making it a safe option [1]. More recently, PDMS has been used in applications such as a reconfigurable frequency selective surface (FSS) for a FabryPerot cavity antenna system [2], bendable and switchable FSS for wearable applications [3], stretchable FSS for high power applications, radomes with mechanical tuning (stretching), electrical tuning for X-band [4] and microfluidic channel based transmit array for linearly-to-circularly-polarized waves using split-ring resonator (SRR) with unit cell rotational capabilities [5].

Satellites are classified based on the distance between the Earth and satellite in space. They are broadly put into strata of the distance a) geostationary earth orbit (GEO), b) medium earth orbit (MEO), and c) low earth orbit (LEO). One of the most widely used LEO satellite systems in recent years is the pico-satellites, which are miniature in size and light in weight (from 0.1 to $1.33 \mathrm{~kg}$ ). The drastic decrease in satellite sizes is spurred by nanotechnology, which decreased the size of the electronic components. The simultaneous increase in launching costs and the need for smaller satellite volume and minimized power consumption has given birth to Cubesatellites (CubeSats). CubeSats are the most common type of pico-satellites [9], [10], and are designed for dedicated tasks such as imaging, wireless body area network (WBAN) localization, remote sensing and deep space communication etc. [6]-[8]; CubeSats can be used for off-body WBAN communications to exchange data from on-body network to PDA then to CubeSat due to its tiny sized $10 \times 10 \times 10 \mathrm{~cm}^{3}$ [9]. A comparison of the mission counts (MC) from the last decade indicates that there is about $80 \mathrm{MC}$ for commercial purposes [11].

Polarization is pivotal when two or more CubeSats need to connect wirelessly with each other, or for the CubeSat to send downlink data to Earth [12]. This is to optimize the limited power on board. The transmitter and receiver should be of the same polarization, otherwise, half of the power will be lost [13], [14]. Most satellites use circular polarization (CP) for this purpose as $\mathrm{CP}$ is capable of penetrating through the earth's atmosphere and has less effect when signals are reflected from the buildings and other natural structures [15], [16]. The design of CP antennas for CubeSat is more complex. Their sizes are typically small, resulting in a low realized gain. This must be compensated using power amplifying circuits on board, which is an unrealistic option for a CubeSat.

An alternative solution to achieving broadband CP is by employing a linear-to-circular polarizer for use in combination with a LP antenna. Available polarizers consist of multilayered rigid substrates, which makes them unsuitable to meet the stringent dimension requirements for CubeSats. A few Sband flexible polarizers exist presently based on textile or PDMS as a substrate, but the size of the unit cell is relatively large [17], [18]. In this paper, a flexible, simple and singlelayered S-Band linear-to-circular polarizing surface is designed and presented. It uses PDMS and compact-sized unit-cells, which maintains ease of fabrication and ease of deployment.

\section{LINEAR TO CIRCULAR POLARIZATION}

\section{A. Conditions}

It is assumed that when an LP wave is impinged on the polarizer with an angle $45^{\circ}$ and travels in the $-z$ direction as shown in Figure 1 and Figure 2, the incident electric field will be decomposed into two electric fields, namely $E_{x}^{i}$ in $x$ direction and $E_{y}^{i}$ in $y$-direction. The ratio of $q$ (between $E_{x}^{i}$ and $E_{y}^{i}$ ) [18], [19]. To simplify analysis, the electric vectors can be rewritten in terms of transmission signals as $T_{x}$ in $x$ direction and $T_{y}$ in $y$-direction, as shown in equation (1) and in Figure 3. To obtain CP wave at the other side of the 
polarizer in $-z$ direction, both electric fields should have equal magnitude but $90^{\circ}$ phase difference between them as shown in (2) and in Figure 4.

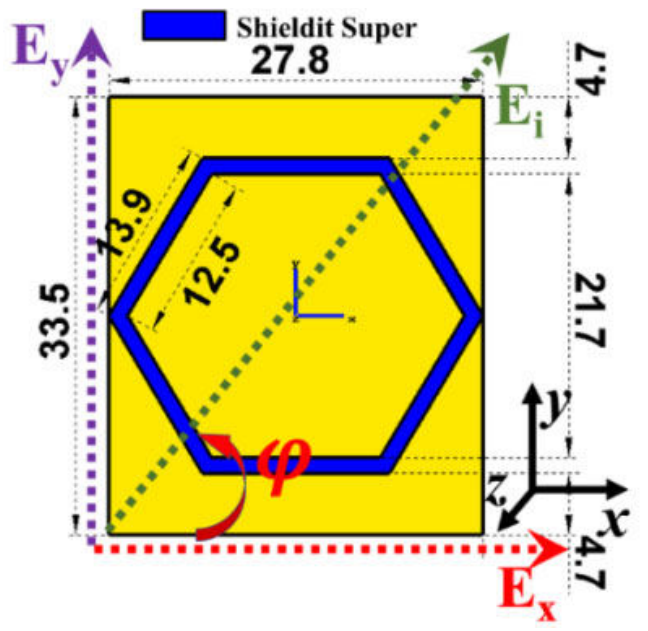

Fig. 1. Front view of the unit cell

PDMS

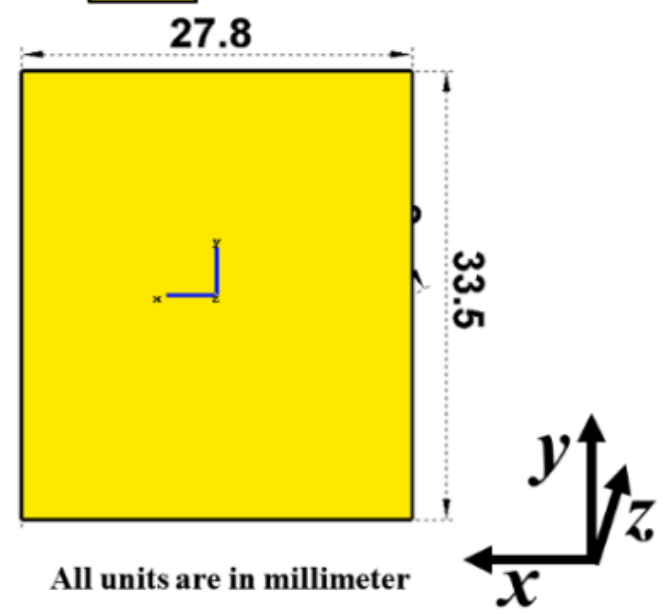

Fig. 2. Back view of the unit cell

$$
\begin{gathered}
\left|E_{x}^{i}\right| \cdot\left|T_{x}\right|=q\left|E_{y}^{i}\right| \cdot\left|T_{y}\right| \\
\Phi\left(T_{x}\right)-\Phi\left(T_{y}\right)=90^{\circ}
\end{gathered}
$$

\section{B. Parameters used for Circular Polarization}

Upon achieving conditions (1) and (2), the value of $q$ will be unity. The $3 \mathrm{~dB}$ axial ratio (AR) is widely accepted in the scientific community to indicate the condition of $\mathrm{CP}$, where a $0 \mathrm{~dB}$ value indicates pure $\mathrm{CP}$. The AR can be calculated using (3), based on the transmission coefficients for $x$ and $y$ polarized waves, respectively. In the figures, the recommended band from FCC [20] is highlighted in blue. The simulated near-zero AR is shown in Figure 5.

To further assess the polarizer, two more parameters are used besides AR: a) conversion efficiency and b) conversion coefficients, which are calculated in (4) and (5), respectively. Parameters $C_{-}$and $C_{+}$are the right-handed circular polarization coefficient and left-handed circular polarization coefficient, as shown in Figure 6 [19], [21].

$$
\begin{gathered}
A R=\sqrt{\frac{\left|T_{y}\right|^{2}+\left|T_{x}\right|^{2}+\left|T_{y}^{2}+T_{x}^{2}\right|}{\left|T_{y}\right|^{2}+\left|T_{x}\right|^{2}-\left|T_{y}^{2}+T_{x}^{2}\right|}} \\
\eta_{c o n v}=\frac{\left(a b s\left(C_{-}\right)^{2}-a b s\left(C_{+}\right)^{2}\right)}{\left(a b s\left(C_{-}\right)^{2}+a b s\left(C_{+}\right)^{2}\right)} \times 100 \\
C_{+}=E_{x}^{i} T_{x}-j E_{y}^{i} T_{y} \\
C_{-}=E_{x}^{i} T_{x}+j E_{y}^{i} T_{y}
\end{gathered}
$$

\section{Unit Cell Design AND Results}

A hexagonal-shaped unit cell is designed and optimized in an electromagnetic simulator. The unit cell boundary condition is defined at the Xmin,Xman,Ymin, and Ymax borders.

The inductive and capacitive creates a phase difference of $90^{\circ}$ between $\Phi_{T_{x}}$ and $\Phi_{T_{y}}$, as shown in Figure 4.

On the other hand, for Z $\min$ and $Z \max$, an open boundary and a Floquet port have been defined, respectively. Shieldit Super conductive fabric is used in place of conventional copper, whereas pure $3 \mathrm{~mm}$-thick PDMS with a dielectric constant $\left(\varepsilon_{r}\right)$ of 2.7 and loss tangent $(\tan \delta)$ of 0.02 is used as its flexible substrate, as shown in Figures 1 and 2 . When an LP wave passes through the polarizing surface, the unit cells connections in the $x$ - direction create an inductive effect in the $E_{i}^{x}$ direction. The gap between the two rows of unit cells creates a capacitive effect for $E_{y}^{i}$ in the $y$ direction, as shown in Figure 7. This is also supported by the high value of surface current which can be observed in between the two adjacent unit cells.

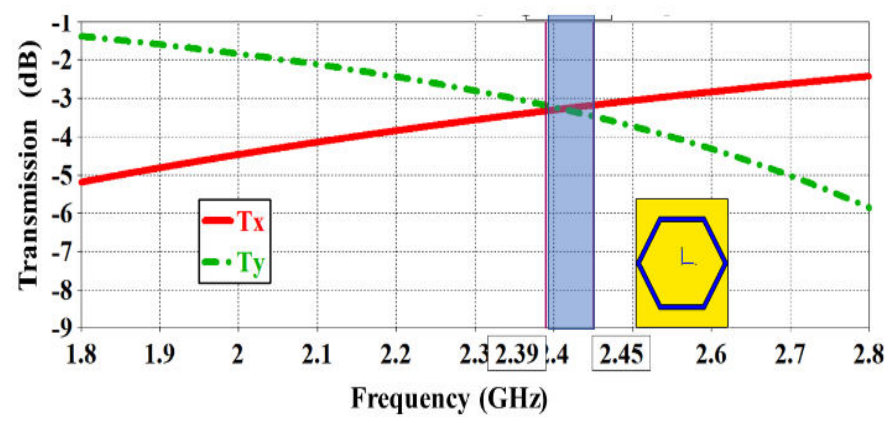

Fig. 3. The transmission coefficient of the two orthogonal components

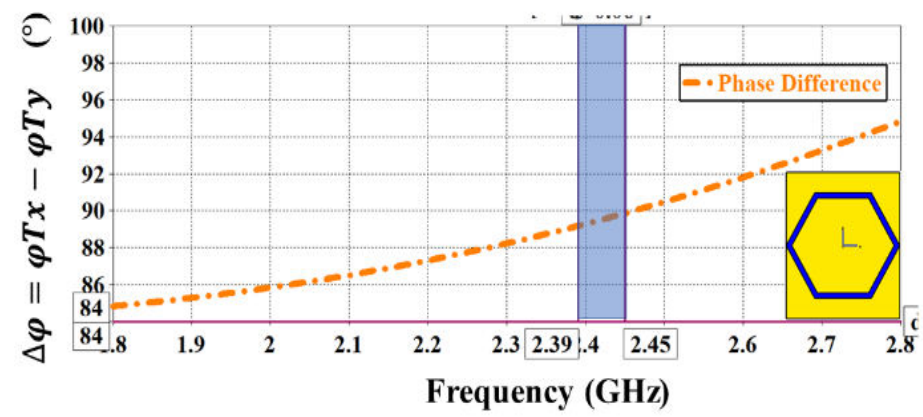

Fig. 4. The phase difference between the two orthogonal components 


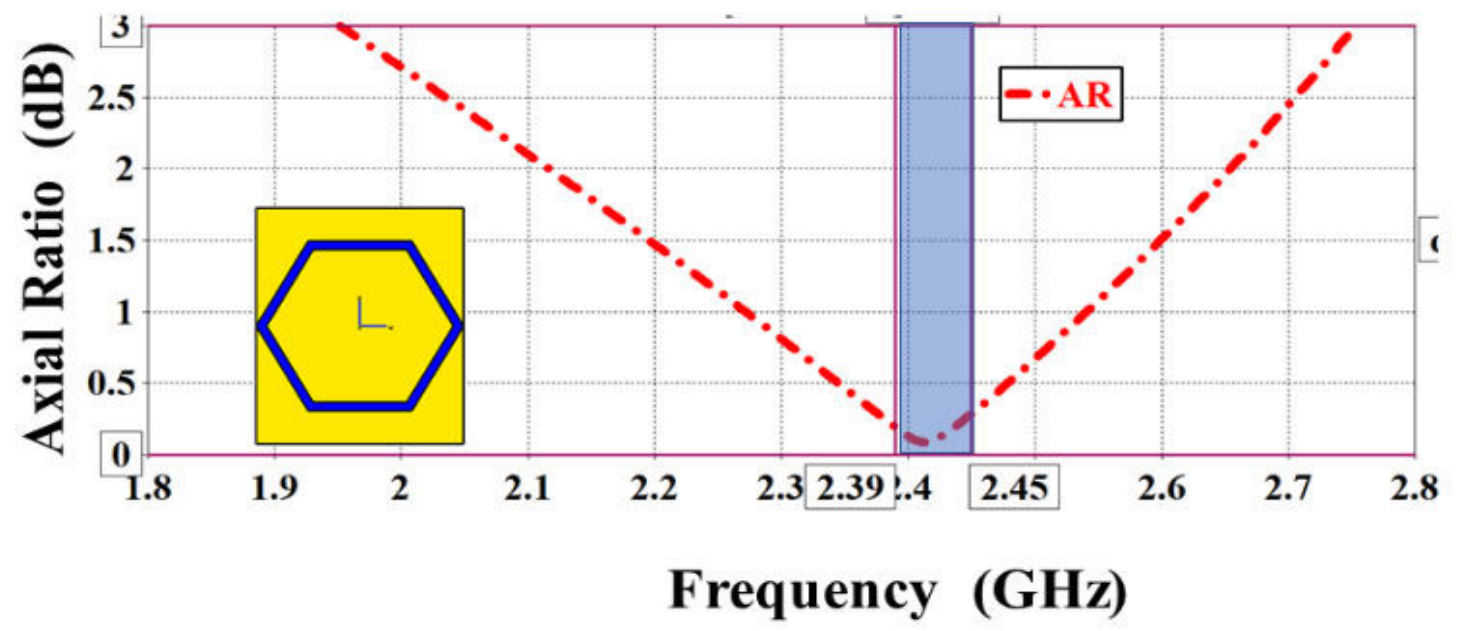

Fig. 5. Axial Ratio
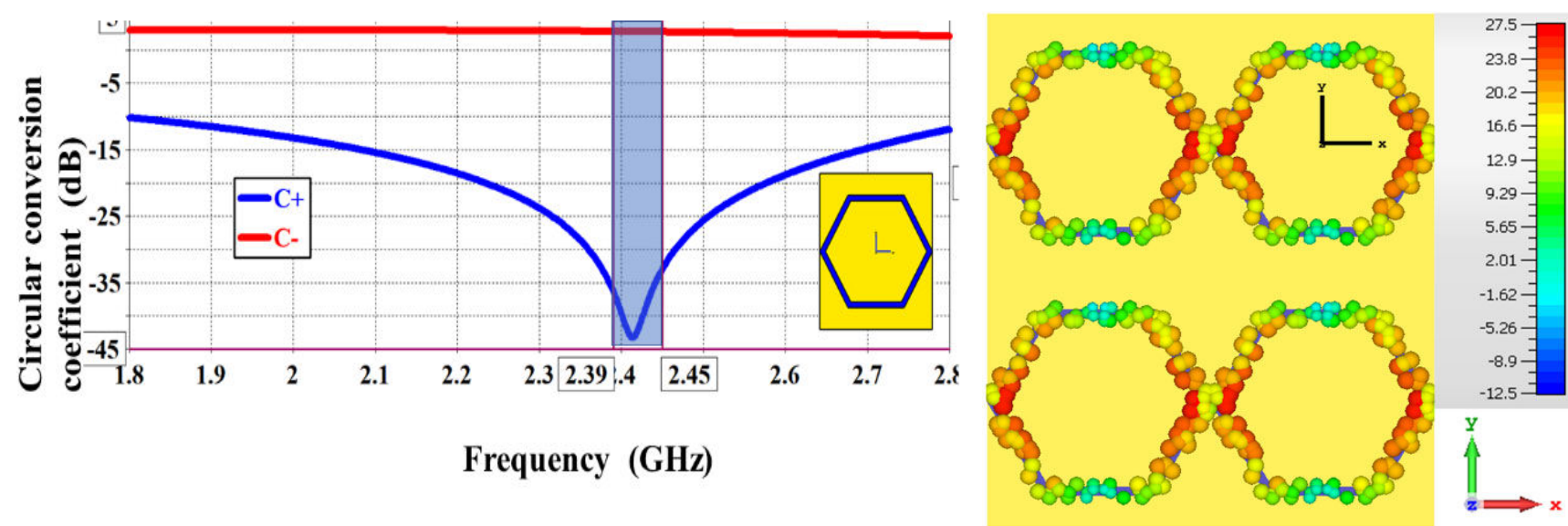

Fig. 6. Conversion coefficient

Fig. 7. The surface current distribution of the unit cells

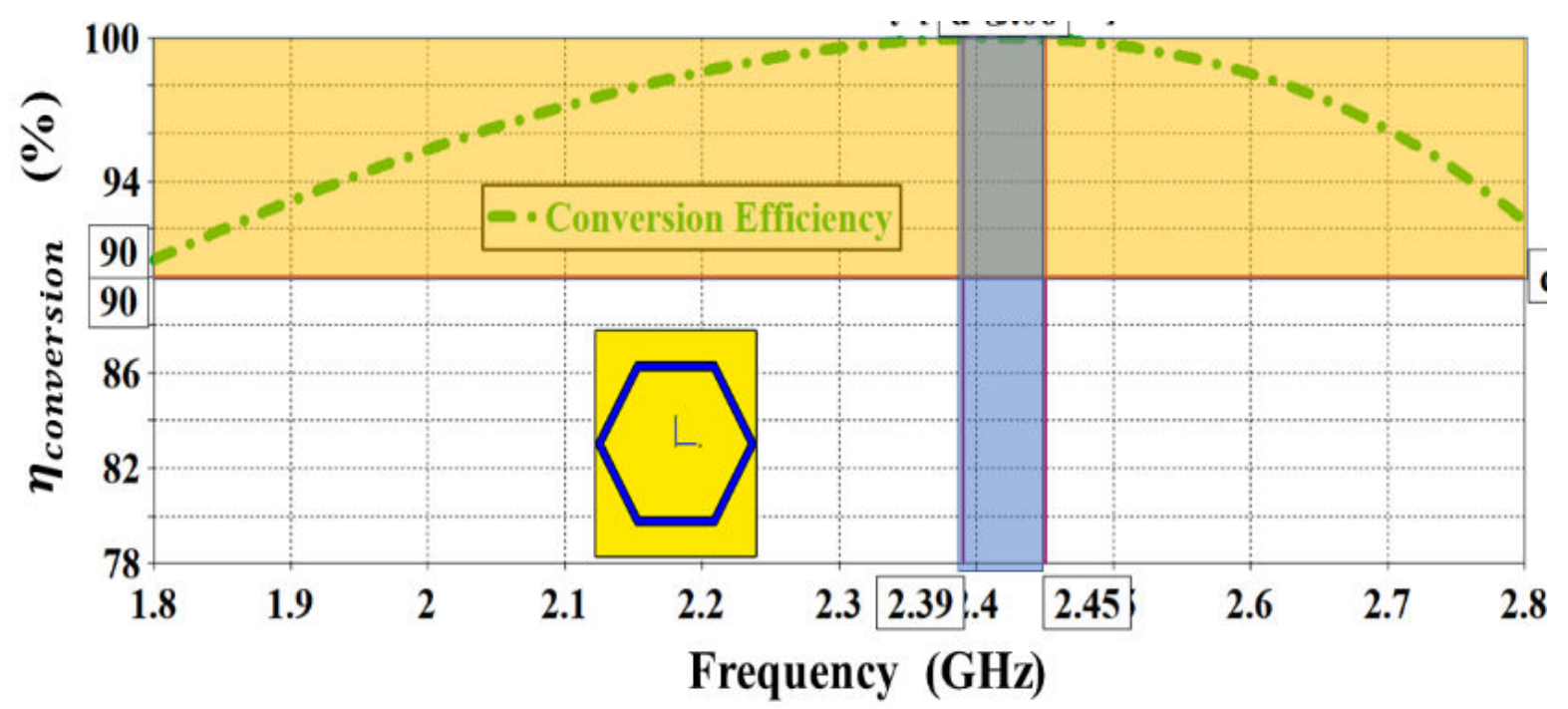

Fig. 8. Conversion efficiency 
The $3 \mathrm{~dB}$ AR of the proposed polarizing surface is shown in Figure 5. Its frequency range starts from $1.95 \mathrm{GHz}$ to 2.76 $\mathrm{GHz}$, with a near-zero minimum value at the center of the FCC recommended S-Band (from 2.39 to $2.45 \mathrm{GHz}$ ) for CubeSat. Finally, the conversion efficiency is shown in Figure 8, with the yellow-highlighted region indicating an efficiency range of $90 \%$. The conversion efficiency above $90 \%$ is from 1.8 $\mathrm{GHz}$ to $2.8 \mathrm{GHz}$, which provides a $43.47 \%$ fractional conversion efficiency bandwidth.

\section{CONCLUSION}

A flexible single-layered and single-sided linear tocircular polarizer has been designed for application in a $3 \mathrm{U}$ CubeSat. Hexagonal-shaped unit cells have been selected for this polarizing surface unit cells to yield the maximum $3 \mathrm{~dB}$ AR fractional bandwidth, besides enabling ease of fabrication. The proposed polarizer has a near-zero axial ratio $(0.08 \mathrm{~dB})$, and its performance assessed using two different approaches (axial ratio and conversion efficiency) resulted in: a) a $3 \mathrm{~dB}$ AR bandwidth of $34.39 \%$ and, b) fractional bandwidth of conversion efficiency (which is $90 \%$ of the maximum value of conversion efficiency) of $43.47 \%$. These values indicate that the polarizer is wideband and can be integrated in CubeSat application in the S-Band for off-body WBAN applications.

\section{ACKNOWLEDGMENT}

This work was supported in part by the Ministry of Higher Education Malaysia (grant no:PRGS/2/2015/ICT06/UNIMAP/02/1) and in part by the Academy of Finland 6Genesis Flagship (grant no. 318927).

\section{REFERENCES}

[1] D. Ponnamma, C. W. Kishor Kumar Sadasivuni, S. Thomas, and M. A.-A. AlMa'adeed, Flexible and Stretchable Electronic Composites. 2016.

[2] C. Lee, R. Sainati, and R. Franklin, "Reconfigurable frequency selective surface for fabry-perot cavity antenna system," 2017 IEEE Antennas Propag. Soc. Int. Symp. Proc., vol. 2017-Janua, pp. 19831984, 2017

[3] H. Zahra, S. M. Abbas, R. M. Hashmi, L. Matekovits, and K. P. Esselle, "Bending analysis of switchable frequency selective surface based on flexible composite substrate," 2019 IEEE Int. Symp. Antennas Propag. Usn. Radio Sci. Meet. APSURSI 2019 - Proc., pp. 2033-2034, 2019.
[4] Y. C. Hung and C. H. Liu, "Stretchable frequency selective surfaces for large-area-tuning and high-power applications,” ISAP 2016 - Int. Symp. Antennas Propag., pp. 942-943, 2017.

[5] E. Erdil, K. Topalli, N. S. Esmaeilzad, O. Zorlu, H. Kulah, and O. Aydin Civi, "Reconfigurable nested ring-split ring transmitarray unit cell employing the element rotation method by microfluidics," IEEE Trans. Antennas Propag., vol. 63, no. 3, pp. 1163-1167, 2015.

[6] R. E. Hodges, N. Chahat, D. J. Hoppe, and J. D. Vacchione, "A Deployable High-Gain Antenna Bound for Mars: Developing a new folded-panel reflectarray for the first CubeSat mission to Mars," IEEE Antennas Propag. Mag., vol. 59, no. 2, pp. 39-49, 2017.

[7] N. Chahat et al., "Advanced CubeSat Antennas for Deep Space and Earth Science Missions: A review,” IEEE Antennas Propag. Mag., vol. 61 , no. 5 , pp. $37-46,2019$.

[8] V. Shirvante, S. Johnson, K. Cason, K. Patankar, and N. G. Fitz-Coy, "Configuration of 3U CubeSat Structures for Gain Improvement of Sband Antennas."

[9] A. H. Lokman et al., "A Review of Antennas for Picosatellite Applications,” Int. J. Antennas Propag., vol. 2017, 2017.

[10] Y. Su, Y. Liu, Y. Zhou, J. Yuan, H. Cao, and J. Shi, "Broadband LEO satellite communications: Architectures and key technologies," IEEE Wirel. Commun., vol. 26, no. 2, pp. 55-61, 2019.

[11] V. Manohar, "For Satellites , Think Small , Dream Big," IEEE Antennas Propag. Mag., no. February 2017, pp. 22-30, 2017.

[12] C. a. Balanis, Antenna Theory: Analysis and Design, vol. 28, no. 3. 2012.

[13] H. Mirza, M. I. Ahmed, and M. F. Elahi, "Circularly polarized compact passive RFID tag antenna,” 2008 Int. Conf. Electr. Comput. Eng., vol. 00, no. December 2008, pp. 20-22, 2008.

[14] H. Mirza and M. F. Elahi, "A UHF-RFID tag antenna for commercial applications,” Proc. ICECE 2008 - 5th Int. Conf. Electr. Comput. Eng., vol. 00, no. December 2008, pp. 764-767, 2008.

[15] B. Y. Toh, R. Cahill, and V. F. Fusco, "Understanding and measuring circular polarization,” IEEE Trans. Educ., 2003.

[16] E. Summary, "Circular Polarization vs . Linear Polarization."

[17] H. Mirza et al., "Deployable Linear-to-Circular Polarizer Using PDMS Based on Unloaded and Loaded Circular FSS Arrays for PicoSatellites,” IEEE Access, vol. 7, pp. 2034-2041, 2019.

[18] H. Mirza et al., "A crossed dodecagonal deployable polarizer on textile and polydimethylsiloxane (PDMS) substrates," Appl. Phys. A Mater. Sci. Process., vol. 124, no. 2, p. 178, 2018.

[19] H. Mirza et al., "Single layered swastika-shaped flexible linear-tocircular polarizer using textiles for S-band application," Int. J. RF Microw. Comput. Eng., vol. 28, no. 7, 2018.

[20] Federal Communications Commission, "Guidance on Obtaining Licenses for Small Satellites," 2013.

[21] H. Mirza, P. J. Soh, A. A. Al-hadi, T. Hossain, and R. Ahmad, "Textilebased flexible linear-to-circular polarizing surface for s- band picosatellites," vol. 8, no. 1, 2019. 